\title{
Band alignment of lateral two-dimensional heterostructures with a transverse dipole
}

O. Leenaerts, S. Vercauteren, and B. Partoens

Citation: Appl. Phys. Lett. 110, 181602 (2017); doi: 10.1063/1.4982791

View online: http://dx.doi.org/10.1063/1.4982791

View Table of Contents: http://aip.scitation.org/toc/apl/110/18

Published by the American Institute of Physics

\section{Articles you may be interested in}

Fano resonance based ultra high-contrast electromagnetic switch

Appl. Phys. Lett. 110, 181904181904 (2017); 10.1063/1.4982725

Thickness-dependent carrier mobility of ambipolar MoTe2: Interplay between interface trap and Coulomb scattering

Appl. Phys. Lett. 110, 183501183501 (2017); 10.1063/1.4982680

Optical evidence for blue shift in topological insulator bismuth selenide in the few-layer limit

Appl. Phys. Lett. 110, 181901181901 (2017); 10.1063/1.4982631

Polarimetric analysis of stress anisotropy in nanomechanical silicon nitride resonators

Appl. Phys. Lett. 110, 181106181106 (2017); 10.1063/1.4982876

Unconventional generation of optical vortex beam using axicon pair and a birefringent lens: Validation of plasmonic excitation

Appl. Phys. Lett. 110, 181105181105 (2017); 10.1063/1.4982875

Widely tunable narrow-linewidth $1.5 \mu \mathrm{m}$ light source based on a monolithically integrated quantum dot laser array

Appl. Phys. Lett. 110, 181103181103 (2017); 10.1063/1.4982716
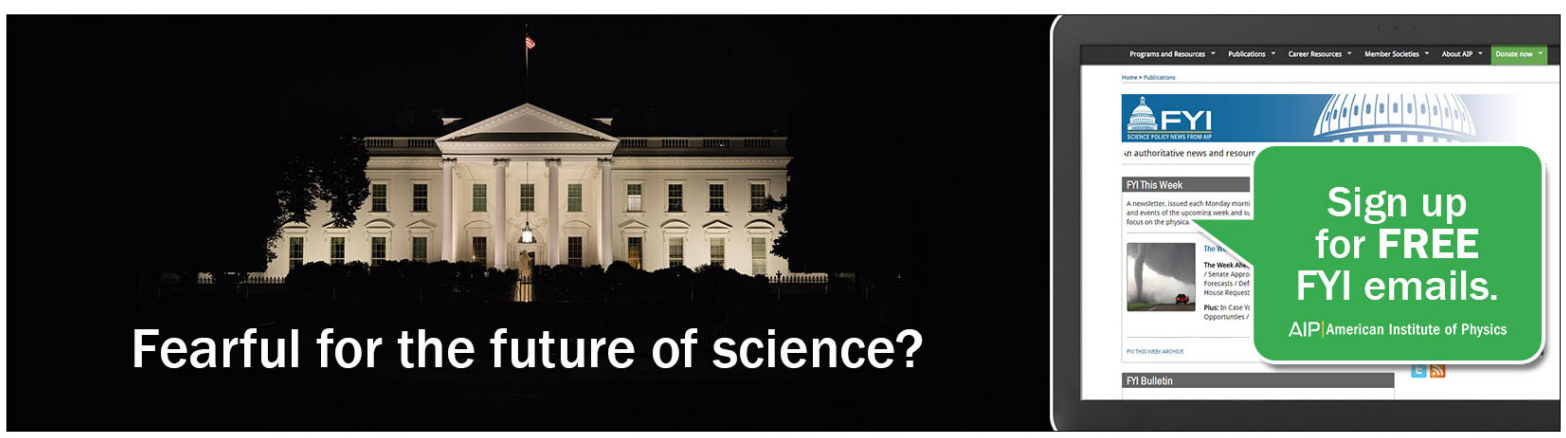


\title{
Band alignment of lateral two-dimensional heterostructures with a transverse dipole
}

\author{
O. Leenaerts, ${ }^{\text {a) }}$ S. Vercauteren, and B. Partoens ${ }^{\text {b) }}$ \\ Universiteit Antwerpen, Departement Fysica, Groenenborgerlaan 171, B-2020 Antwerpen, Belgium
}

(Received 24 January 2017; accepted 19 April 2017; published online 1 May 2017)

\begin{abstract}
It was recently shown that the electronic band alignment in lateral two-dimensional heterostructures is strongly dependent on the system geometry, such as heterostructure width and layer thickness. This is so even in the absence of polar edge terminations because of the appearance of an interface dipole between the two different materials. In this study, this work is expanded to include two-dimensional materials that possess an electronic dipole over their surface, i.e., in the direction transverse to the crystal plane. To this end, a heterostucture consisting of polar hydrofluorinated graphene and non-polar graphane layers is studied with first-principles calculations. As for nonpolar heterostructures, a significant geometry dependence is observed with two different limits for the band offset. For infinitely wide heterostructures, the potential step in the vacuum is equally divided over the two sides of the heterostructure, resulting in a finite potential step in the heterostructure. For infinitely thick heterostructure slabs, on the other hand, the band offset is reduced, similar to the three-dimensional case. Published by AIP Publishing.
\end{abstract}

[http://dx.doi.org/10.1063/1.4982791]

The experimental realization of heterostructures from two-dimensional (2D) materials such as graphene and hexagonal boron nitride ${ }^{1-6}$ has stimulated the theoretical study of such systems tremendously. Stacked heterostructures, which result from stacking different 2D materials layer by layer on top of each other, have been well studied by now. ${ }^{7-9}$ The interaction among the different layers is usually of the van der Waals-type in that case. But 2D heterostructures can also be formed through covalent in-plane connections between different sheets. ${ }^{10-12}$ In the latter case, special care is needed to calculate the electronic band alignment. An important aspect is the possibility of polar discontinuities at the edge/ interface of 2D polar materials or grain boundaries within polycrystalline samples. ${ }^{13-16}$ In those cases, an electrostatic instability can arise that leads to edge metallization or the adsorption of ionic groups at the edge. But even in the absence of in-plane polarity, some complications show up at the interface of lateral 2D heterostructures. At first sight, the absence of intrinsic electric dipoles seems to imply the validity of Anderson's rule which states that the vacuum potential can be used as a fixed reference level for the band alignment. ${ }^{17}$ However, it has been recently demonstrated ${ }^{18,19}$ that there exists an interface dipole at the heterojunction of any two 2D materials which leads to a band alignment that depends on the specific geometry of the heterostructure. If the lateral dimensions of the system are small enough, substantial steps in the vacuum potential above the heterojunction can be observed.

Here, we expand this previous work to $2 \mathrm{D}$ materials with two inequivalent surfaces. The different structure or composition of the surfaces leads to a net electric dipole over the material. The 2D material therefore forms a dipole layer that divides space into two regions with a different

\footnotetext{
a) ortwin.leenaerts@uantwerpen.be

b)bart.partoens@uantwerpen.be
}

vacuum potential. When such a polar layer is combined with a non-polar layer into a lateral heterostructure, it is not immediately clear how their respective bands should be aligned. Even for an infinitely wide heterostructure, it is not possible to align the vacuum levels of the two materials according to Anderson's rule because there are two different vacuum levels for the polar material.

In our study, we make use of hydrofluorinated few-layer graphene (HFG) which consists of a graphene multilayer that is fully covered with hydrogen atoms on one side and with fluorine atoms on the other. The strong covalent bonds with the adsorbates turn the graphene multilayer to a thin diamond film. ${ }^{20-23}$ Due to the different electron affinity of $\mathrm{H}$ and $\mathrm{F}$, a strongly polar layer is formed. The hydrofluorinated graphene is subsequently combined with a fully hydrogenated graphene (HG) sample (i.e., graphane). An example of such a heterostructure is shown in Figure 1. The reason for this specific choice of artificial materials is fourfold: (i) They are both wide-gap semiconductors, avoiding various extra effects due to possible charge transfers and (ii) their similar lattice constants make covalent in-plane connections possible with negligible strain. ${ }^{24}$ (iii) They also share the same diamond-like 3D bulk limit for thick multilayered systems and (iv) have one surface (the hydrogenated one) in common while the other is very different. These specific features turn out to facilitate the understanding of the band alignment and allow us to understand more complex systems. Note that although we use the HG/HFG heterostructure as a convenient model, there is some theoretical ${ }^{20-23,25-28}$ and experimental ${ }^{29-33}$ support that such systems could be made.

We performed first-principles calculations within the density functional theory methodology as implemented in the OpenMX code. Norm-conserving pseudopotentials and pseudo-atomic localized basis functions ${ }^{34-36}$ are used. More precisely, a double-valence plus single polarization orbital basis set is used for all atoms. The exchange-correlation 


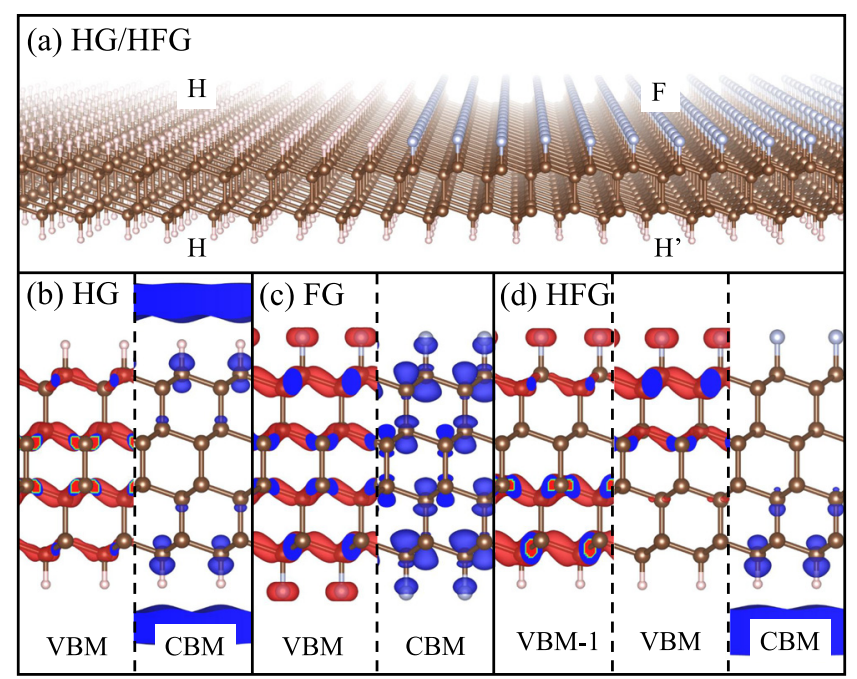

FIG. 1. (a) HG/HFG heterostructure model for a slab with two carbon layers. (b) and (c) The density of the electron states corresponding to the VBM, VBM-1, and the CBM of graphane, fluorographane, and hydrofuorographene, respectively.

functional is approximated with the generalized gradient method as described by Perdew, Burke, and Ernzerhof ${ }^{37}$ (PBE). Calculations for isolated graphane and hydrofluorographene are done within unit cells containing two carbon atoms per atomic layer and a $12 \times 12 \times 1 k$-point grid for the selfconsistent cycles. More accurate density of states calculations are computed using a $33 \times 33 \times 1 k$-point mesh. HG/HFG heterostructures are simulated with supercells of varying sizes from 16 to 48 unit cells (u.c.) and up to several hundred carbon atoms per layer. As for the isolated systems, $1 \times 12 \times 1$ and $1 \times 33 \times 1 k$-point meshes are used for the self-consistent cycle and density of states calculations, respectively. To avoid interlayer interaction normal to the slabs, we make use of the effective screening method (ESM), ${ }^{38}$ as implemented in OpenMX. In this method, the periodic boundary conditions are broken in one direction and can be replaced by vacuum conditions. Previous calculations have shown that the results of this method are consistent with those of very large vacuum regions and periodic boundary conditions. ${ }^{18}$

Before studying 2D heterostructures of graphane and hydrofluorographene, we first take a closer look at the isolated systems. For comparison, we also consider fully fluorinated graphene (FG), known as fluorographene (FG). These three functionalized graphene slabs are all semiconductors with similar diamond-like $\mathrm{C}$ interiors but different surface compositions. While HG and FG have two equivalent surfaces, HFG has not and consequently exhibits a strong polarity in the out-of-plane direction. The density of the charge carriers at the band edges is shown in Figs. 1(b)-1(d) for $\mathrm{HG}$, $\mathrm{FG}$, and HFG slabs containing four $\mathrm{C}$ layers. The state corresponding to the valence band maximum (VBM) of $\mathrm{HG}$ and FG appears to be localized in the bulk, while the conduction band maximum (CBM) is a surface state for $\mathrm{HG}$ and a bulk state for FG. In fact, the FG VBM and CBM states are also surface states, but this feature becomes only apparent for thicker slabs (see supplementary material, Fig. S1). The CBM state of HFG is localized at the $\mathrm{H}$ surface and is similar to the CMB of HG. The VBM of HFG, on the other hand, is localized at the F surface and resembles the VBM of FG. The state below the VBM of HFG, denoted as VBM-1, is more localized in the bulk with the bulk-like character increasing as the number of layers is increased (see supplementary material, Fig. S1). This state is similar to the VBM state of HG. Because the CBM states of HG and HFG are surface states, the VBM (and VBM-1) is more suited to study the band alignment in this work. Therefore, we characterize the band positions through the ionization energy (IE), defined as the difference in energy between the vacuum level and the VBM.

The IEs of HG, FG, and HFG are plotted as a function of the number of layers in Fig. 2. For HFG, two IEs can be defined because the dipole over the system creates two different vacuum levels. Therefore, we plot the difference between the two and compare it to the difference between the IE of graphane and fluorographene. For thick slabs, these differences seem to converge but they never become equal because the energy of the FG-like surface state of HFG converges to a value of about $0.1 \mathrm{eV}$ above the bulk VBM, as shown in the supplementary material (Fig. S2). The IE for the $\mathrm{H}$ side of $\mathrm{HFG}$ will consequently never converge to the IE of $\mathrm{HG}$, because it is defined with respect to a surface state above the bulk VBM. Therefore, it could be more meaningful to define the IE of $\mathrm{HFG}$ at the $\mathrm{H}$ side with respect to the bulk VBM, i.e., VBM-1, so that a thick HFG slab can be regarded as the superposition of half a $\mathrm{HG}$ and half a FG slab. To avoid these complications, we focus on variations in the vacuum potential from which the band positions can be unambiguously deduced.

Next, we combine HG and HFG into a lateral heterostructure. An example with two $\mathrm{C}$ layers is shown in Fig. 1(a), but we focus first on a monolayer system. To study the band alignment, the differences in vacuum potential on both sides of the heterojunction, above and below the heterostructure, are calculated. These differences are referred to as $\Delta \mathrm{V}_{\mathrm{vac}}^{\mathrm{HF}}$ and $\Delta \mathrm{V}_{\mathrm{vac}}^{\mathrm{HH}^{\prime}}$ (see Fig. 3) at the fluorinated and hydrogenated sides of HFG, respectively, with the labels $\mathrm{H}, \mathrm{H}^{\prime}$, and F defined in Fig. 1(a).

The vacuum potentials below and above a monolayer $\mathrm{HG} / \mathrm{HFG}$ heterostructure are shown in Fig. 4. Away from the

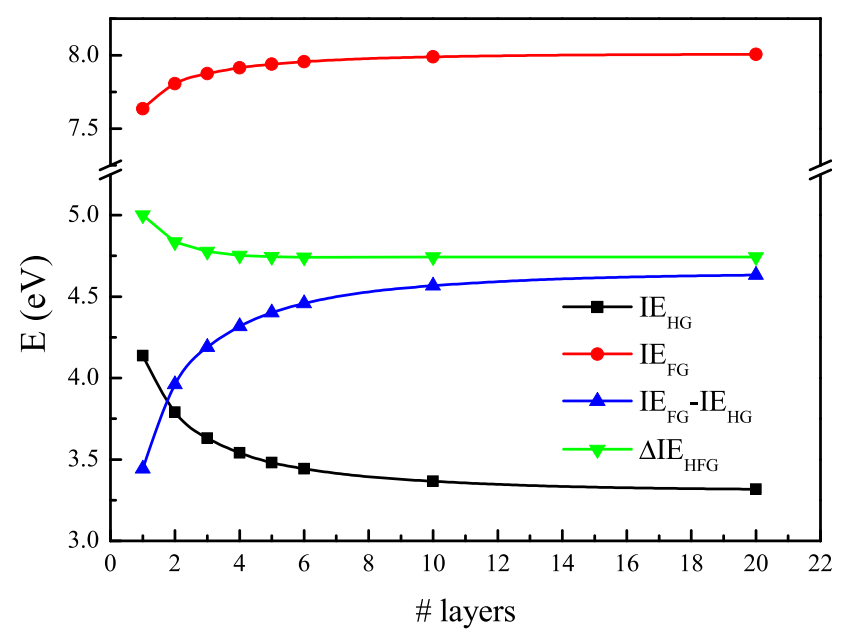

FIG. 2. The evolution of the ionization energies of $\mathrm{HG}$ and FG as a function of the number of layers, together with the difference between the two and the potential difference over the HFG layer. 


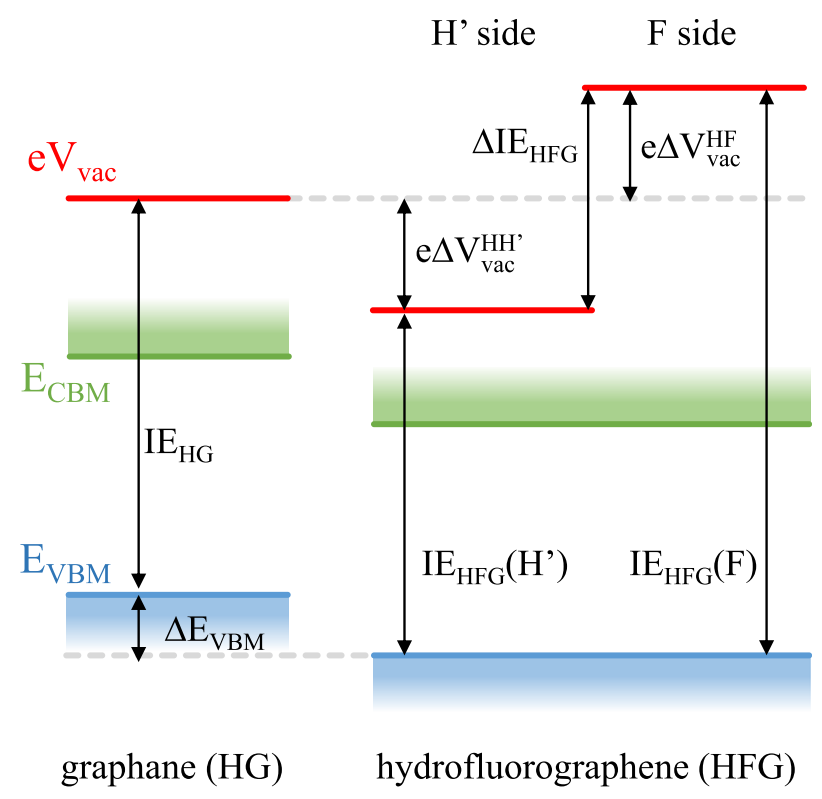

FIG. 3. Schematic drawing of the important energy levels in graphane and hydrofluorographene. The arrows indicate the energy differences as defined in the text.

interface, the potential at the HF side converges rapidly and the difference between the two sides is well defined. On the $\mathrm{HH}^{\prime}$ side, the potential converges much slower because of the similar electron states at that side which can easily intermix. ${ }^{24}$ The potential difference is calculated as the difference between the vacuum potential at the middle of the two materials. For convenience, we define the potential step as a positive quantity, i.e., $\Delta \mathrm{V}_{\text {vac }}^{\mathrm{HF}}=\mathrm{V}_{\text {vac }}^{\mathrm{F}}-\mathrm{V}_{\text {vac }}^{\mathrm{H}}$ and $\Delta \mathrm{V}_{\text {vac }}^{\mathrm{HH}^{\prime}}=\mathrm{V}_{\text {vac }}^{\mathrm{H}}-\mathrm{V}_{\text {vac }}^{\mathrm{H}^{\prime}}$.

As observed before, ${ }^{18}$ the potential step in the vacuum depends on the width of the heterostructure. Therefore, we investigate the variation of $\Delta \mathrm{V}_{\mathrm{vac}}$ as a function of inverse heterostructure width (Fig. 5). For comparison, we also show the valence band alignment as calculated from the atomprojected density of states at the center of the slab on both sides of the heterostructure. With increasing width, the potential steps in the vacuum above and below the heterostructure

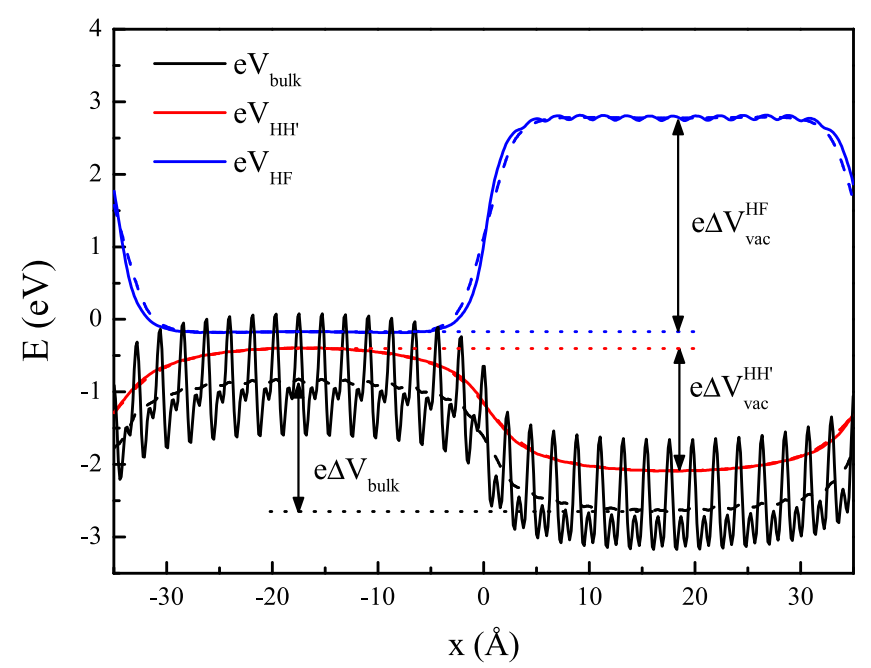

FIG. 4. The potential in the vacuum below $\left(\mathrm{HH}^{\prime}\right)$, above $(\mathrm{HF})$, and inside a monolayer HG/HFG heterostructure with a width of 16 u.c. The dashed lines represent lattice averaged potentials.

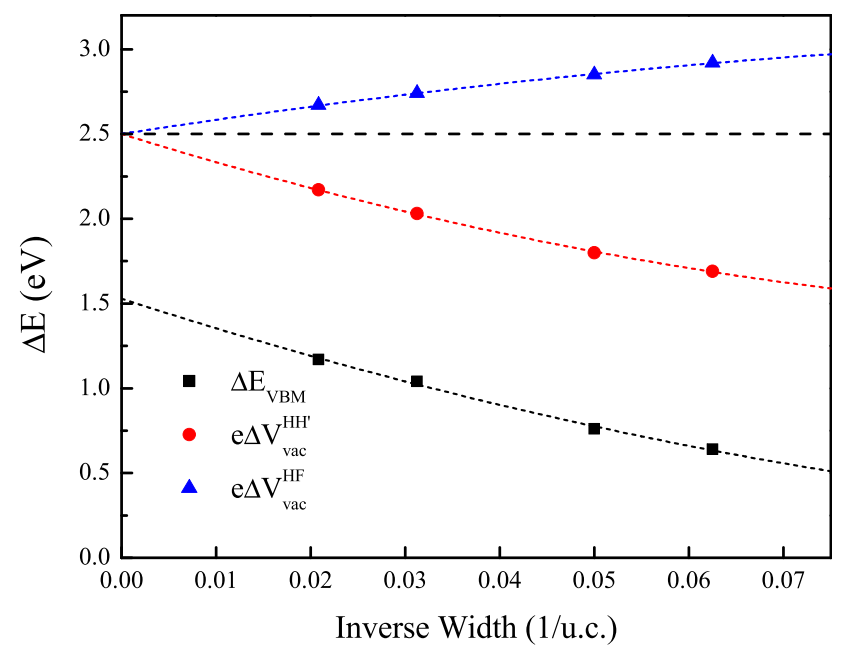

FIG. 5. The difference in vacuum potential below $\left(\mathrm{HH}^{\prime}\right.$, red) and above $(\mathrm{HF}$, blue) the heterostructure and the band offset (black) as a function of inverse heterostructure width for a monolayer system.

are seen to converge to the same value. This value of approximately $2.5 \mathrm{eV}$ equals half the difference in IE between the two sides of HFG (see Fig. 2). Therefore, we can conclude that the vacuum step due to the polarity of HFG is equally divided between both sides of the heterostructure for infinitely wide heterostructures.

As a final study of the geometry dependence of polar heterostructures, we investigate the variation of the vacuum potential step as a function of heterostructure thickness at constant width. It is convenient to take the ratio of the potential steps from the two sides of the heterostructure. As seen in Fig. 6, this ratio depends linearly on the heterostructure with a slope that decreases with increasing heterostructure width. Two different limiting situations can be constructed from this figure, as schematically depicted in Fig. 7. First, the ratio of the potential steps for a heterostructure with a given thickness converges to one as the width increases, as was also explicitly shown for the monolayer case in Fig. 5. Second, for infinite heterostructure thickness, the ratio becomes infinite indicating that the vacuum levels at the $\mathrm{H}$ side are aligned.

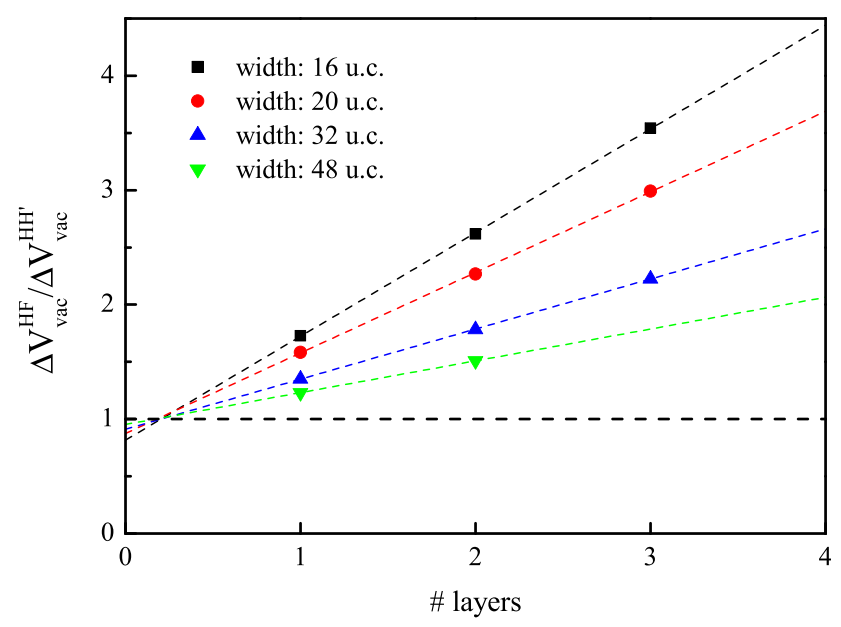

FIG. 6. Ratio of vacuum steps from both sides of the heterostructures as a function of slab thickness for different widths. 

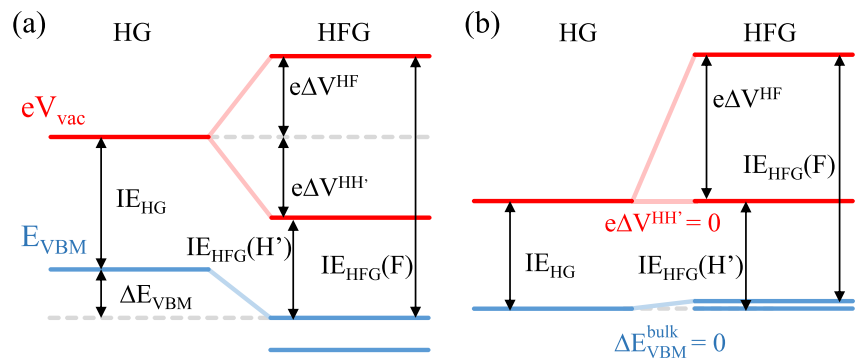

FIG. 7. Schematic representation of the band and vacuum level alignment of a HG/HFG heterostructure of infinite width (a) and infinite thickness (b). Why the lowest level determining $\operatorname{IE}_{\mathrm{HFG}}\left(\mathrm{H}^{\prime}\right)$ is different in (a) an (b) is discussed in Section I of the supplementary material.

As recently shown in the literature, the dependence of the band alignment on the geometry (width and thickness) of 2D lateral heterostructures is a consequence of the emergence of an interface dipole at the heterojunction between two 2D materials. ${ }^{18,19}$ For a periodic array of slabs, the band alignment changes linearly with the inverse heterostructure width. ${ }^{19}$ This behavior is also seen for the polar heterostructures in our work, although a slight nonlinearity can be observed in Fig. 5. For the limit of an infinite heterostructure width, the influence of the interface dipole vanishes and the vacuum potential step is equally divided over the two sides of the heterostructure, as can be understood from basic electrostatics (see supplementary material, Fig. S3). This observation is easily generalized to heterostructures made of two polar crystals. The potential steps in the vacuum determine the band alignment in the bulk of the heterostructure and can lead to a band offset. This band offset can induce a charge polarization (interface dipole) at the heterojunction, similar to heterostructures in $3 \mathrm{D},{ }^{39-43}$ which tends to reduce the band offset. Contrary to the 3D case, the interface dipole has finite range in $2 \mathrm{D}$ and gives rise to the observed geometry dependence. As the 2D heterostructure slab thickness increases, the total interface dipole grows until the (3D) bulk band alignment is reached. In our special case, the bulk regions of the two sides of the heterostructure were chosen to be the same (i.e., diamond) so that the band offset in the bulk disappears.

In this study, we expanded previous work on the band alignment of lateral 2D heterostructures to include twodimensional materials that possess an electronic dipole over their surface. We made use of a well-chosen model heterostructure consisting of non-polar graphane and polar hydrofluorinated graphene layers. Similar to non-polar heterostructures, a significant geometry dependence is observed with two distinct limits for the band offset. Infinitely wide heterostructures have potential steps in the vacuum that are equally divided over the two sides of the heterostructure. This leads to a finite potential step in the heterostructure and, consequently, an interface dipole. This interface dipole becomes dominant for infinitely thick heterostructure slabs, resulting in a reduced band offset, similar to the 3D case. For the studied HG/HFG heterostructure, the band offset completely vanishes because of the equal bulk limits of HG and HFG. Note that this is not a trivial result because the different surface dipoles are still present in the system.
See supplementary material for further details on the electron states corresponding to the band extrema of HFG and their edge/bulk character. We also provide a schematic picture of the electrostatic potential due to polarized surfaces in a slab.

This work was supported by the Fonds Wetenschappelijk Onderzoek (FWO-Vl). The computational resources and services used in this work were provided by the VSC (Flemish Supercomputer Center), funded by the Hercules Foundation and the Flemish Government-department EWI.

${ }^{1}$ L. Ci, L. Song, C. Jin, D. Jariwala, D. Wu, Y. Li, A. Srivastava, Z. F. Wang, K. Storr, L. Balicas, F. Liu, and P. M. Ajayan, Nat. Mater. 9, 430 (2010).

${ }^{2}$ M. P. Levendorf, C.-J. Kim, L. Brown, P. Y. Huang, R. W. Havener, D. A. Muller, and J. Park, Nature 488, 627 (2012).

${ }^{3}$ L. Liu, J. Park, D. A. Siegel, K. F. McCarty, K. W. Clark, W. Deng, L. Basile, J. C. Idrobo, A.-P. Li, and G. Gu, Science 343(6167), 163 (2014).

${ }^{4}$ X. Duan, C. Wang, J. C. Shaw, R. Cheng, Y. Chen, H. Li, X. Wu, Y. Tang, Q. Zhang, A. Pan, J. Jiang, R. Yu, Y. Huang, and X. F. Duan, Nat. Nanotechnol. 9, 1024 (2014).

${ }^{5}$ Y. Gong, J. Lin, X. Wang, G. Shi, S. Lei, Z. Lin, X. Zou, G. Ye, R. Vajtai, B. I. Yakobson, H. Terrones, M. Terrones, B. K. Tay, J. Lou, S. T. Pantelides, Z. Liu, W. Zhou, and P. M. Ajayan, Nat. Mater. 13, 1135 (2014).

${ }^{6}$ C. Huang, S. Wu, A. M. Sanchez, J. J. P. Peters, R. Beanland, J. S. Ross, P. Rivera, W. Yao, D. H. Cobden, and X. Xu, Nat. Mater. 13, 1096 (2014).

${ }^{7}$ A. K. Geim and I. V. Grigorieva, Nature 499, 419 (2013).

${ }^{8}$ L. Britnell, R. M. Ribeiro, A. Eckmann, R. Jalil, B. D. Belle, A. Mishchenko, Y.-J. Kim, R. V. Gorbachev, T. Georgiou, S. V. Morozov, A. N. Grigorenko, A. K. Geim, C. Casiraghi, A. H. Castro Neto, and K. S. Novoselov, Science 340, 1311 (2013).

${ }^{9}$ C.-H. Lee, G.-H. Lee, A. M. van der Zande, W. Chen, Y. Li, M. Han, X. Cui, G. Arefe, C. Nuckolls, T. F. Heinz, J. Guo, J. Hone, and P. Kim, Nat. Nanotechnol. 9, 676 (2014).

${ }^{10}$ Q. Sun, Y. Dai, Y. Ma, N. Yin, W. Wei, L. Yu, and B. Huang, 2D Mater. 3, 035017 (2016).

${ }^{11}$ H. Jin, J. Li, B. Wang, Y. Yu, L. Wan, F. Xu, Y. Dai, Y. Wei, and H. Guo, J. Mater. Chem. C 4, 11253 (2016).

${ }^{12}$ Y. Guo and J. Robertson, Appl. Phys. Lett. 108, 233104 (2016).

${ }^{13}$ J. M. Pruneda, Phys. Rev. B 81, 161409(R) (2010).

${ }^{14}$ N. C. Bristowe, M. Stengel, P. B. Littlewood, E. Artacho, and J. M. Pruneda, Phys. Rev. B 88, 161411 (2013).

${ }^{15}$ M. Gibertini, G. Pizzi, and N. Marzari, Nat. Commun. 5, 5157 (2014).

${ }^{16}$ R. Martinez-Gordillo and M. Pruneda, Prog. Surf. Sci. 90, 444 (2015).

${ }^{17}$ R. L. Anderson, Solid-State Electron. 5, 341 (1962).

${ }^{18}$ O. Leenaerts, S. Vercauteren, B. Schoeters, and B. Partoens, 2D Mater. 3, 025012 (2016).

${ }^{19}$ J. Zhang, W. Xie, J. Zhao, and S. Zhang, 2D Mater. 4, 015038 (2017).

${ }^{20}$ O. Leenaerts, B. Partoens, and F. M. Peeters, Phys. Rev. B 80, 245422 (2009).

${ }^{21}$ L. Zhu, H. Hu, Q. Chen, S. Wang, J. Wang, and F. Ding, Nanotechnol. 22, 185202 (2011).

${ }^{22}$ J. Sivek, O. Leenaerts, B. Partoens, and F. M. Peeters, J. Phys. Chem. C 116, 19240 (2012).

${ }^{23}$ D. Odkhuu, D. Shin, R. S. Ruoff, and N. Park, Sci. Rep. 3, 3276 (2013).

${ }^{24}$ M. N. Amini, O. Leenaerts, B. Partoens, and D. Lamoen, J. Phys. Chem. C 117, 16242 (2013).

${ }^{25}$ J. O. Sofo, A. S. Chaudhari, and G. D. Barber, Phys. Rev. B 75, 153401 (2007).

${ }^{26}$ O. Leenaerts, H. Peelaers, A. D. Hernández-Nieves, B. Partoens, and F. M. Peeters, Phys. Rev. B 82, 195436 (2010).

${ }^{27}$ H. Sahin, O. Leenaerts, S. K. Singh, and F. M. Peeters, Wiley Interdiscip. Rev.: Comput. Mol. Sci. 5, 255 (2015).

${ }^{28}$ A. Kvashnin, L. A. Chernozatonskii, B. I. Yakobson, and P. B. Sorokin, Nano Lett. 14, 676 (2014).

${ }^{29}$ D. C. Elias, R. R. Nair, T. M. G. Mohiuddin, S. V. Morozov, P. Blake, M. P. Halsall, A. C. Ferrari, D. W. Boukhvalov, M. I. Katsnelson, A. K. Geim, and K. S. Novoselov, Science 323, 610 (2009).

${ }^{30}$ S. H. Cheng, K. Zou, F. Okino, H. R. Gutierrez, A. Gupta, N. Shen, P. C. Eklund, J. O. Sofo, and J. Zhu, Phys. Rev. B 81, 205435 (2010). 
${ }^{31}$ F. Withers, M. Dubois, and A. K. Savchenko, Phys. Rev. B 82, 073403 (2010).

${ }^{32}$ R. R. Nair, W. C. Ren, R. Jalil, I. Riaz, V. G. Kravets, L. Britnell, P. Blake, F. Schedin, A. S. Mayorov, S. Yuan, M. I. Katsnelson, H. M. Cheng, W. Strupinski, L. G. Bulusheva, A. V. Okotrub, I. V. Grigorieva, A. N. Grigorenko, K. S. Novoselov, and A. K. Geim, Small 6, 2877 (2010).

${ }^{33}$ S. Rajasekaran, F. Abild-Pedersen, H. Ogasawara, A. Nilsson, and S. Kaya, Phys. Rev. Lett. 111, 085503 (2013).

${ }^{34}$ T. Ozaki, Phys. Rev. B 67, 155108 (2003).

${ }^{35}$ T. Ozaki and H. Kino, Phys. Rev. B 69, 195113 (2004).
${ }^{36}$ T. Ozaki and H. Kino, Phys. Rev. B 72, 045121 (2005).

${ }^{37}$ J. P. Perdew, K. Burke, and M. Ernzerhof, Phys. Rev. Lett. 77, 3865 (1996).

${ }^{38}$ M. Otani and O. Sugino, Phys. Rev. B 73, 115407 (2006).

${ }^{39}$ F. Flores and C. Tejedor, J. Phys. C 12, 731 (1979).

${ }^{40}$ J. Tersoff, Phys. Rev. B 30, 4874 (1984).

${ }^{41}$ J. Tersoff, Phys. Rev. B 32, 6968 (1985).

${ }^{42}$ J. Robertson, J. Vac. Sci. Technol., A 31, 050821 (2013).

${ }^{43}$ Y. Hinuma, A. Grüneis, G. Kresse, and F. Oba, Phys. Rev. B 90, 155405 (2014). 